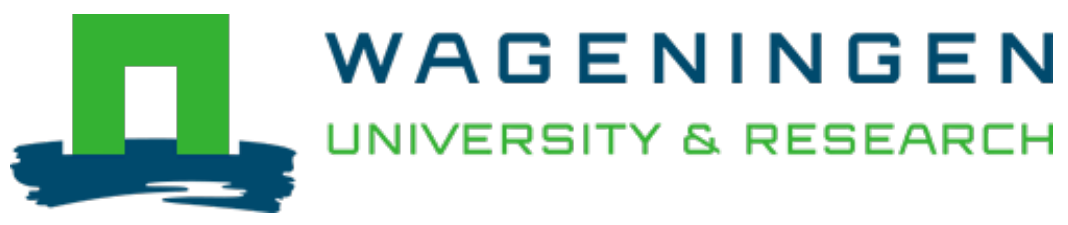

\title{
Direct and quantitative in-situ analysis of third-hand smoke in and on various matrices by ambient desorption corona beam ionization mass spectrometry
}

Talanta

Min, Ke; Guo, Ping; Chen, Dongying; Huang, Si; Luo, Wei et al

https://doi.org/10.1016/j.talanta.2020.121330

This publication is made publicly available in the institutional repository of Wageningen University and Research, under the terms of article $25 \mathrm{fa}$ of the Dutch Copyright Act, also known as the Amendment Taverne. This has been done with explicit consent by the author.

Article 25 fa states that the author of a short scientific work funded either wholly or partially by Dutch public funds is entitled to make that work publicly available for no consideration following a reasonable period of time after the work was first published, provided that clear reference is made to the source of the first publication of the work.

This publication is distributed under The Association of Universities in the Netherlands (VSNU) 'Article $25 \mathrm{fa}$ implementation' project. In this project research outputs of researchers employed by Dutch Universities that comply with the legal requirements of Article $25 \mathrm{fa}$ of the Dutch Copyright Act are distributed online and free of cost or other barriers in institutional repositories. Research outputs are distributed six months after their first online publication in the original published version and with proper attribution to the source of the original publication.

You are permitted to download and use the publication for personal purposes. All rights remain with the author(s) and / or copyright owner(s) of this work. Any use of the publication or parts of it other than authorised under article $25 \mathrm{fa}$ of the Dutch Copyright act is prohibited. Wageningen University \& Research and the author(s) of this publication shall not be held responsible or liable for any damages resulting from your (re)use of this publication.

For questions regarding the public availability of this publication please contact openscience.library@wur.nl 


\title{
Direct and quantitative in-situ analysis of third-hand smoke in and on various matrices by ambient desorption corona beam ionization mass spectrometry
}

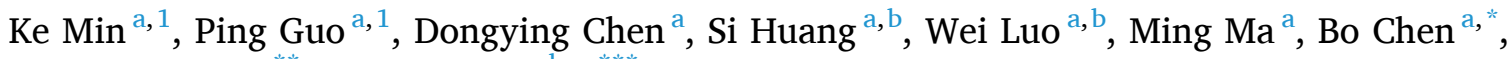 \\ Shouzhuo Yao ${ }^{\text {a, } * *}$, Han Zuilhof ${ }^{\text {a, b, c, }{ }^{* * * *}}$ \\ ${ }^{a}$ Key Laboratory of Phytochemical R\&D of Hunan Province, Key Laboratory of Chemical Biology \& Traditional Chinese Medicine Research of Ministry of Education, \\ Hunan Normal University, Changsha, 410081, China \\ ${ }^{\mathrm{b}}$ Laboratory of Organic Chemistry, Wageningen University, Stippeneng 4, 6703 WE, Wageningen, the Netherlands \\ ${ }^{\mathrm{c}}$ Department of Chemical and Materials Engineering, Faculty of Engineering, King Abdulaziz University, Jeddah, Saudi Arabia
}

\section{A R T I C L E I N F O}

\section{Keywords:}

Third-hand smoke

Desorption corona beam ionization

Ambient mass spectrometry

In-situ analysis

Surface analysis

Tobacco-specific nitrosamines

\begin{abstract}
A B S T R A C T
Third-hand smoke (THS) is composed of surface-deposited remnants resulting from tabacco-smoking. Because THS components have properties of remaining on, re-emitting from and reacting on and with surfaces, in-situ analysis of the components on different surfaces is both in high demand and challenging. The aim of this study is to establish desorption corona beam ionization (DCBI)-MS/MS as an analytical tool for THS research. To this end, an in-situ DCBI-MS/MS approach was developed for the quantitative analysis of typical THS environmental markers, i.e. nicotine and cotinine on different surfaces such as fruits, cotton clothing, glass, and toys etc. The limits of detection of nicotine and cotinine were both $1.4 \mu \mathrm{g} \mathrm{m}^{-2}$. Low-temperature DCBI-MS/MS was applied to the direct detection of THS on fingers without any skin damage. Smoking-related biomarkers analyses in urine were accomplished, with a 10 s DCBI analysis time. The on-surface tobacco-specific nitrosamines (TSNAs), such as 1-(N-methyl-N-nitrosamino)-1-(3-pyridinyl)-4-butanal) (NNA), 4-(methylnitrosamino)-1-(3-pyridinyl)-1butanone (NNK), and N-nitroso nornicotine (NNN) were in-situ successfully detected in dust samples.
\end{abstract}

\section{Introduction}

The worldwide average use of cigarettes is still above 1000 cigarettes per year for persons of 14 and older. This number decreases locally in some countries, due to governmental regulations, but globally continues to reflect a serious health risk, also given the continued starting of smoking habits among young adults in still growing populations. Thirdhand smoke (THS), a term first coined in 2006 [1], refers to the pollution that persists after secondhand smoke (SHS) is released into the air. It refers to the gases and particles released from the burning tobacco that are embedded on or in materials such as carpets, furniture, clothes, toys, skin and fruits [2]. Strictly speaking, THS is not smoke, but the chemicals attached to the surface that can be released back into the air through chemical transformation and/or accumulation $[3,4]$, or be taken up by the human body upon contact with THS-contaminated surfaces.

In recent years, although a large number of studies have demonstrated the potential health effects of THS exposure, such as cytotoxicity, changes in metabolism, cellular structure damage, damage to the liver, lungs, skin, and deviant behavior (of mice), the awareness of these risks in the general population is still low $[4,5]$. The hazards of THS as public health problem are far less well documented than those of smoking itself or SHS, urging further studies of THS and resulting ailments.

Unlike SHS, THS specifically includes residual surface contamination from tobacco smoke adsorption, the re-volatilization of adsorbed pollutants on the surfaces into the gas phase, and the formation of new toxic substances from the reaction of pollutants with oxidants or other compounds in the environment (e.g., ozone and nitrous acid (HONO)) [6-8].

\footnotetext{
* Corresponding author.

$* *$ Corresponding author.

$* * *$ Corresponding author. Laboratory of Organic Chemistry, Wageningen University, Stippeneng 4, 6703 WE, Wageningen, the Netherlands.

E-mail addresses: dr-chenpo@vip.sina.com (B. Chen), SZYao@hnu.cn (S. Yao), han.zuilhof@wur.nl (H. Zuilhof).

1 These authors contributed equally.
} 
For example, residual nicotine absorbed from tobacco smoke onto indoor surfaces reacts with HONO present in household and outdoor environments to form cancer-causing tobacco-specific nitrosamines (TSNAs), i.e. 1-(N-methyl-N-nitrosamino)-1-(3-pyridinyl)-4-butanal) (NNA), 4-(methylnitrosamino)-1-(3-pyridinyl)-1-butanone (NNK), and $\mathrm{N}$-nitroso nornicotine (NNN). These chemicals are widely found in unburned tobacco and tobacco smoke, and display carcinogenic effects [6]. Thus, exposure to THS components involves three typical processes, namely remaining, re-emitting and reacting [3]. Due to this presence, release and reaction of THS components on widely differing surfaces, the in-situ analysis of THS components on these different surfaces is of great significance to the study of THS.

In a systematic review of THS literature in 2018 [5], 30 papers analyzed the nicotine content in THS, 17 focused on nitrosamines, and 15 analyzed cotinine. In general, the most commonly used biomarkers for THS were nicotine, nitrosamine and cotinine. This indicates that these compounds are the main targets for the qualitative and quantitative study of THS. Currently, the most commonly used method for detecting third-hand smoke contamination is to analyze the concentration of nicotine and cotinine in the environment by indirect wipe-sampling $[9,10]$, but this method requires a complicated and time-consuming sample preparation, specifically when comparisons need to be made between highly different surfaces (ranging from silicone rubber via pears and peaches to plastic bottles). Therefore, direct in-situ analytical methods for THS research are in urgent need [11].

Ambient ionization MS (AMS) refers to "the ionization of unprocessed or minimally modified samples in their native environment, and it typically refers to the ionization of condensed phase samples in air" [12]. Due to the typical characteristics of AMS, it significantly speeds up the in situ analysis of blood, tissue, cell culture and other biological samples, as well as the study of surface analysis such as that of surface monolayer modification [13-16] and mass spectrometric imaging [17-22]. AMS has also been used in research on tobacco smoke exposure. For example, paper spray ionization (PSI) was used for the quantitative analysis of nicotine and cotinine in cell cultures exposed to tobacco smoke, to investigate the applicability of PSI as a tool for in vitro cytotoxicity testing [23]. Direct analysis in real-time (DART)-MS and DART-MS/MS were used to detect THS and monitor SHS. Tobacco smoke contamination (THS) on the clothing of smokers and the transfer of tobacco smoke contamination from the fingers of smokers to other objects were detected [24]. However, it would also be of significant interest to develop an AMS-based method to measure directly on skin. Specifically, the determination of nicotine on skin, especially of fingers, is of interest, as it is a likely indicator of overall (contact-based) exposure to THS [25]. For example, when measured by the wipe method, skin nicotine concentrations reached values up to $1160 \mathrm{ng} /$ wipe in smokers [26], while for non-smokers exposure to SHS, exposure to THS and non-exposure yielded up to 49, 46 and $18 \mathrm{ng}$ /wipe [27,28]. However, e. g. DART is based on thermal desorption mechanisms, and for bio-surfaces such as fingertips, and skin in general, working at high desorption temperatures is, of course, out of the question due to burning and current-induced problems. Despite these highly useful contributions by AMS to THS research, the quantitative in situ surface analysis of THS, the direct THS analysis of biologically relevant surfaces, and the study of THS reaction products, remain extremely challenging for AMS.

Desorption corona beam ionization (DCBI) for AMS was proposed in 2010 [29]. Although it has a similar ionization mechanism with DART, there are some significant differences. First, DCBI makes use of a hollow needle/annular electrode structure to form a thin and optically visible corona beam of about $0.1 \mathrm{~cm}$ wide and $1 \mathrm{~cm}$ long (Fig. S1), which greatly facilitates the positioning and imaging analysis of the sampling area. Second, the DCBI source can provide a programmed temperature increase from room temperature to $450{ }^{\circ} \mathrm{C}$, so as to achieve a separate and controllable desorption of components in the sample, and thereby reduce the complexity of resulting mass spectra. AMS analysis of various compounds in a wide variety of sample substrates has been successfully performed using a DCBI source [30-36]. For cigarette analysis, DCBI-MS successfully characterized the spatial distribution of phenol in an acetate fiber tip [37]. In addition, the capture of intermediates by DCBI-MS directly verified the Leuckart reaction mechanism, which also indicated that DCBI is an effective tool for the detection of intermediates in some thermal reactions [38].

Here, we present the development of DCBI-MS/MS into an analytical tool for THS research. To this end, quantitative in-situ DCBI-MS/MS analysis was investigated of typical THS environmental markers, such as nicotine and cotinine, on a wide variety of different household-based surfaces, such as fruits, cotton clothing, glass, rubber nipples, toys, etc. (see Fig. 1). In addition, a method was developed to directly analyze skin (finger tips). In this we analyze both the effects of standard burning cigarettes, as well as that of heat-not-burn cigarettes - often advertised as healthier - in which the smoker inhales an aerosol resulting from gently heating tobacco in a battery-driven device, rather than actually burn the tobacco. To demonstrate the potential of DCBI-MS/MS for biomarker detection, a straightforward urine analysis was accomplished. Finally, to investigate a series THS reactions as happening on surfaces, various tobacco-specific nitrosamines (TSNAs) in dust samples were directly and in-situ analyzed.

\section{Experimental}

\subsection{Chemicals and materials}

All solvents (HPLC-grade) were purchased from Merck (Darmstadt, Germany). Deionized water was obtained using a Milli-Q ${ }^{\mathrm{TM}}$ Direct $\mathrm{ul}-$ trapure water system (Millipore, USA). Nicotine, and cotinine standards were from Sigma-Aldrich, (Steinheim, Germany). Nicotine- $\mathrm{d}_{4}$ was from Zhenzhun Co. (Shanghai, China), and functioned as internal standard (I. S.). NNA, NNK, NNN were purchased from Sigma (St. Louis, MO, USA). Glycerin and chloroform were analytical grade reagents purchased from Sinopharm (Beijing, China). All samples including fresh fruits, toys, infant milk bottles, etc. were purchased from local supermarkets (Changsha, China). The samples were washed by ultrapure water and were prior to the subsequent exposure to smoke - shown to be nicotinefree, as detected by DCBI-MS/MS at $90{ }^{\circ} \mathrm{C}$ desorption temperature. Human urine was obtained from the affiliated hospital of Hunan Normal University.

\subsection{Instrumentation and conditions}

All experiments were performed using a Shimadzu DCBI-1 ionization source coupled to a LCMS-8040 mass spectrometer (Shimadzu, Japan) working in multiple reaction monitoring (MRM) mode and in positive ion mode. MS/MS characteristic ion pair were performed as the following transitions: $m / z 163.1$ to $m / z 130.1$ for nicotine, $m / z 167.1$ to $\mathrm{m} / \mathrm{z} 134.1$ for nicotine- $\mathrm{d}_{4}, \mathrm{~m} / \mathrm{z} 177.1$ to $\mathrm{m} / \mathrm{z} 80.0$ for cotinine, $\mathrm{m} / \mathrm{z} 208.1$ to $m / z 177.1$ for NNA, $m / z 208.2$ to $m / z 122.1$ for NNK, and $m / z 178.1$ to $m / z 120.1$ for NNN. The flow rate of helium (He), high voltage (HV) discharge, and gas temperature (T) were set at different values depending on sample characteristics, as specified in Table S1. The conditions of the MS interface, controlled by a LabSolutions workstation, were as follows: desolvation line (DL) temperature $250{ }^{\circ} \mathrm{C}$, heated block temperature $300{ }^{\circ} \mathrm{C}$, collision-induced dissociation (CID) gas pressure $230 \mathrm{kPa}$, collision energy (CE) $22 \mathrm{~V}$. The other instruments involved in this study include a desktop high-speed centrifuge (model TG16G; Hunan Kaida Co., Changsha, China), electronic microbalance (model AP135W; Shimadzu, Japan), and Finn pipettes (Thermo Fisher Scientific, Finland).

\subsection{In-situ surface quantitative analysis of THS}

\subsubsection{THS simulation}

A plastic box $(1.0 \mathrm{~m} \times 1.0 \mathrm{~m} \times 1.0 \mathrm{~m}, \mathrm{~L} \times \mathrm{W} \times \mathrm{H})$ with a small door 


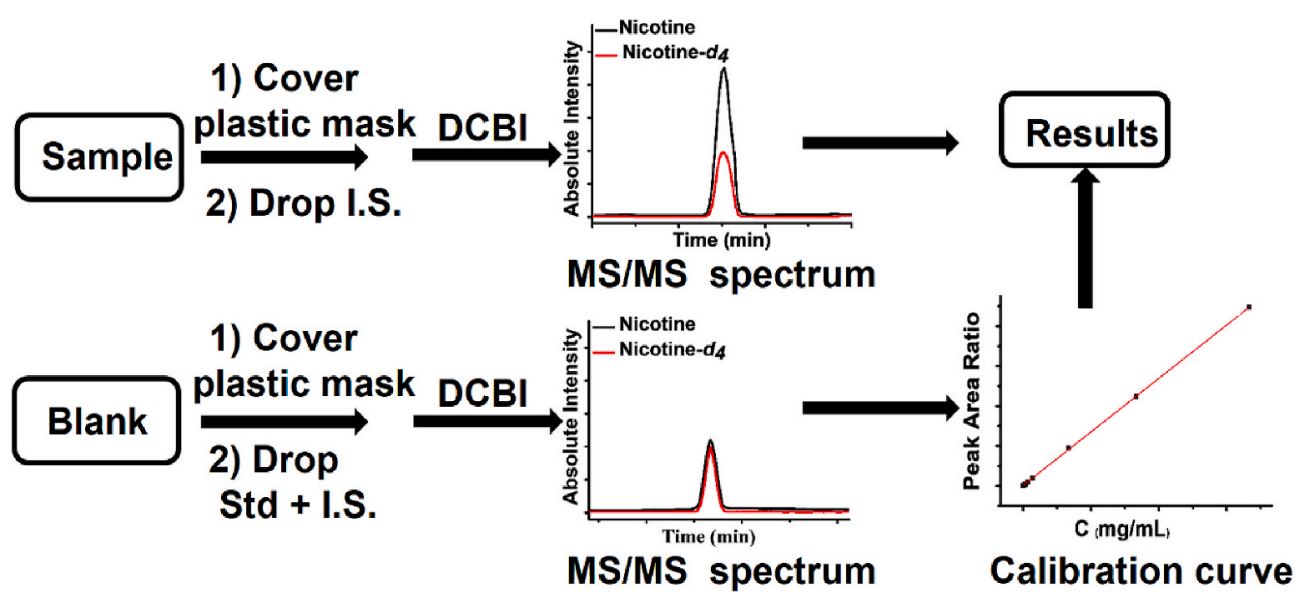

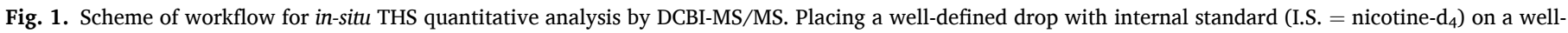

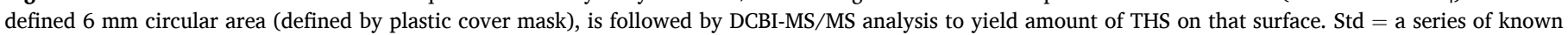
concentrations standard solution of nicotine and cotinine.

on one side was used as THS formation chamber, as depicted in Fig. S2, two small electric fans, which were used to provide a constant steady airflow $(0.1 \mathrm{~m} / \mathrm{s})$ throughout the entire experiment, were placed at one corner of the bottom and on the diagonal of the top in the box, respectively. Constant airflow velocity was confirmed prior to the experiment with an anemometer (multichannel anemometer model 1560, Kanomax, Inc., Andover, NJ, USA) at three locations i.e. the top, middle and bottom of the chamber. We confirmed that the air inside was well mixed in the chamber during the smoking deposition.

Before a variety of samples being put into the THS formation chamber, we tested a standard cigarette burning in dry air $\left(32{ }^{\circ} \mathrm{C}\right)$, natural burnout takes about $8 \mathrm{~min}$. So a lit cigarette was placed at the center of the chamber for $10 \mathrm{~min}$ and it was ensured that it fully burned out in each deposition experiment, and then gently removed from the test chamber (with minimal opening to the outside). In addition, after the cigarette was removed, the smoking deposition time on sample surfaces was estimated in a closed THS formation chamber. The results (see Fig. S3) showed that both the relative signal intensity of nicotine absorbed on the four different surfaces (i.e., cherry tomato, cotton clothing, glass and filter paper) was enough to reach a relatively stable value at deposition time $20 \mathrm{~min}$. So the chamber was kept in the closed state for $20 \mathrm{~min}$ after the cigarette was removed to let any atmospheric compounds deposit on any available surfaces (of either the chamber walls or of objects placed in the chamber). Then these samples were taken out and covered with plastic adhesive tape (termed plastic mask) with a round hole (diameter $6 \mathrm{~mm}$ ) (see Fig. S4a of photograph of an example); this plastic mask with a $6 \mathrm{~mm}$ diameter hole was made by a custom-build handheld holing machine, and the area of the hole was about $0.28 \mathrm{~cm}^{2}$, as depicted in Fig. S4b. Finally, the plastic maskcovered samples were analyzed by DCBI-MS/MS.

\subsubsection{Quantitative in-situ surface analysis}

The sample surface was covered by a plastic mask with a round hole (diameter $6 \mathrm{~mm}$; see Fig. 1) with a resulting area of about $0.28 \mathrm{~cm}^{2} .2 \mu \mathrm{L}$ of a $0.5 \mathrm{mg} \mathrm{mL}^{-1}$ nicotine- $\mathrm{d}_{4}$ (I.S) acetonitrile solution was dropped on the center of sample surface, which was exposed in the hole. After evaporation of the solvent ( 2 min under ambient conditions; no air flow was used), the surface was analyzed under the DCBI corona beam. For the construction of calibration curves (all in acetonitrile) of nicotine and cotinine, series of standard working solutions (including $0.5 \mathrm{mg} \mathrm{L} \mathrm{L}^{-1}$ nicotine- $\mathrm{d}_{4}$ as internal standard) of nicotine $(0.05,0.1,0.5,1.0,10,25$, $\left.50 \mathrm{mg} \mathrm{L}^{-1}\right)$ and cotinine $\left(0.05,0.1,0.5,1.0,5.0,10,20 \mathrm{mg} \mathrm{L}^{-1}\right)$ were prepared as follows: $100 \mathrm{mg} \mathrm{L}^{-1}$ of both nicotine and cotinine acetonitrile solutions were used as standard storage solutions. Different volume of nicotine and cotinine standard solutions, as well as different volume of $100 \mathrm{mg} \mathrm{L}^{-1}$ nicotine- $\mathrm{d}_{4}$ (I.S.) acetonitrile solution was added in the mixtures. Then each of these mixtures was diluted with acetonitrile to a fixed volume, thus obtaining a series of standard solutions of nicotine and cotinine. For measurements, $2.0 \mu \mathrm{L}$ of these standard solutions was dropped on $0.28 \mathrm{~cm}^{2}$ blank surface from four different sample, e.g. cherry tomato, cotton clothing, glass and filter paper; these samples were then allowed to dry prior to analysis by DCBI-MS/MS. The calibration curves were constructed by plotting the signal peak area ratio $(Y)$ of target ion to internal standard ion versus the concentration of target ion $(X)$.

\subsection{Biomarker analysis of THS in urine}

Urine samples of 52 adults and 14 babies (six newborn babies and eight 3 9-month old babies) were obtained from the affiliated hospital of Hunan Normal University. Obtaining and testing these urine samples was approved by the Hunan Normal University Ethics Committee. The adults' urine samples were treated as follow: $100 \mu \mathrm{L}$ of urine was mixed with $400 \mu \mathrm{L}$ of acetonitrile solution containing $400 \mathrm{ng}$ nicotine- $\mathrm{d}_{4}$, then vortexed for $5 \mathrm{~min}$. After centrifugation, $5.0 \mu \mathrm{L}$ of supernatant was dropped at the tip of a thin sampling glass capillary (see Fig. S5a), which was then allowed to dry in air before analysis by DCBI-MS/MS. In order to enrich two THS biomarkers (nicotine and cotinine) in infant urine samples, the dispersive liquid-liquid micro-extraction (DLLME) method was used. To this aim, $0.5 \mathrm{~g}$ of solid $\mathrm{NaCl}$ was added to $5 \mathrm{~mL}$ of urine in a plug centrifugal tube, and the $\mathrm{pH}$ was then adjusted to 9 by addition of 1 $\mathrm{mol} \mathrm{L}^{-1} \mathrm{NaOH}$ solution. After the $\mathrm{NaCl}$ dissolved, a mixture of $200 \mu \mathrm{L}$ of chloroform (including $1 \mathrm{mg} \mathrm{L}^{-1}$ nicotine- $\mathrm{d}_{4}$ ) as an extractant, and 1000 $\mu \mathrm{L}$ of methanol as a dispersing agent were added to the urine solution. Then the mix solution was vortexed for $2 \mathrm{~min}$, and subsequently centrifuged for $5 \mathrm{~min}$ at $4000 \mathrm{rpm}$. $5 \mu \mathrm{L}$ of the lower organic phase solution was analyzed by DCBI-MS/MS.

\subsection{Direct and in-situ monitoring of TSNAs}

For direct quantitative analysis of TSNAs in indoor dust, samples were obtained from hotel rooms in which it was allowed to smoke, and the analysis mode was similar with that of biomarker analysis of THS in urine. Dust was collected at the tip of a sampling glass capillary rod (see Fig. S5b) that was coated with a thin glycerin film. The sample weight was obtained by comparing the weight difference before and after sampling using an electronic microbalance. Subsequently, 5.0 $\mu \mathrm{L}$ of acetonitrile solution containing $0.8 \mu \mathrm{g} \mathrm{mL}^{-1}$ nicotine- $\mathrm{d}_{4}$ was dropped in the dust sample at the tip of sampling glass capillary rod, and let it dry in air before analysis by DCBI-MS/MS. A series of solutions of equimolar 
amounts of NNA, NNK, NNN (each in the range $0.1-50 \mathrm{mg} \mathrm{L}^{-1}$ ) were made, to which was added $0.8 \mu \mathrm{g} \mathrm{mL}^{-1}$ nicotine- $\mathrm{d}_{4}$ (I.S.). $5.0 \mu \mathrm{L}$ of these standard solutions was dropped on a sampling glass capillary rod coated with glycerin film, left to dry, and then analyzed by DCBI-MS/MS. Then the calibration curves were constructed by using the signal peak area ratio $(Y)$ of target ion to internal standard ion to the concentration of target ion $(X)$.

\section{Results and discussion}

\subsection{In-situ surface quantitative analysis of THS}

First, we investigate the possibility of DCBI for the quantitative insitu analysis of THS-contaminated surfaces (expressed in $\mu \mathrm{g} \mathrm{m}^{-2}$ ). In this approach, all contaminants in a definite area are analyzed at once in a direct manner. The detection area is, in principle, determined by the size of the DCBI corona beam spot at the surface. We investigated the most effective area for the quantitative detection by DCBI (working at $1.0 \mathrm{~L} \mathrm{~min}^{-1}$ flow rate of $\mathrm{He}, 1.5 \mathrm{kV}$ high voltage discharge, and $50{ }^{\circ} \mathrm{C}$ gas temperature) by using a glass surface onto which nicotine was uniformly deposited in the THS simulation chamber as a model surface. Masks with a round hole with a diameter from 2 to $15 \mathrm{~mm}$ were then used to define the exposed surface; $2 \mu \mathrm{L}$ of I.S. solution (nicotine- $\mathrm{d}_{4}$ ) was added in the middle of the hole. When the diameter is larger than $8 \mathrm{~mm}$ (hole area $\geq 0.5 \mathrm{~cm}^{2}$ ), the deposited nicotine on the surface in the hole could not be fully evaporated fast enough to be analyzed in a quantitative manner, which leads to deviations from linearity of calibration curves $\left(\mathrm{R}^{2}<\right.$ 0.982). When the diameter was $\leq 6 \mathrm{~mm}$ (hole' area about $0.28 \mathrm{~cm}^{2}$ ), the peak positions and peak widths of nicotine and I.S. overlapped well; in this situation $6 \mathrm{~mm}$ holes are preferable, as they displayed the optimum between quantification and signal/noise (S/N) ratio. Typical DCBI-MS/ MS peak shapes of nicotine and nicotine- $\mathrm{d}_{4}$ on holes of 6 and $15 \mathrm{~mm}$ diameters respectively that were passed in front of the detector sequentially were shown in Fig. 2.

Using these $6 \mathrm{~mm}$ diameter holes, calibration curves and limits of detection (LODs; determined at $\mathrm{S} / \mathrm{N}=3$ ) were constructed for nicotine and cotinine on four different surfaces such as cherry tomato, cotton clothing, glass and filter papers (see Table S2). From the calibration curves, in all cases excellent linearity was observed (all $\mathrm{R}^{2}$ was greater than 0.994 , and closed to 0.999 for most), and the LODs of nicotine and cotinine $\left(\mathrm{S} / \mathrm{N}=3\right.$ ) were both found to be $1.4 \mu \mathrm{g} \mathrm{m}^{-2}$; typical data on filter paper see Fig. 3, Fig. 3a clearly showed the unambiguous detection of protonated nicotine at $m / z 163.1$ and protonated cotinine at $m / z 177.1$, which were identified by MS/MS experiment (Fig. 3b and c). Fig. 3d and e shows excellent linear relation. Based on these, we selected filter paper as a model matrix to construct the calibration curve to quantitatively

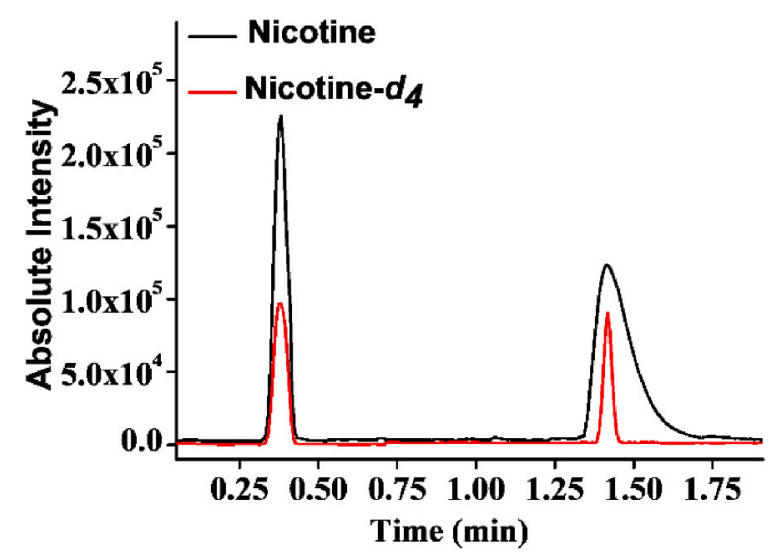

Fig. 2. DCBI-MS/MS peak shapes of uniformly deposited nicotine and nicotine$\mathrm{d}_{4}$ dropped in the middle of a $6 \mathrm{~mm}$ hole (peak at $0.25-0.50 \mathrm{~min}$ ) and a $15 \mathrm{~mm}$ hole (1.25-1.75 $\mathrm{min})$. determine THS on the various different surfaces under investigation. The results are presented in Table 1 . Different from any wiping-based method that needs to quantitatively remove the analyte from the surface to be analyzed, the in-situ DCBI-MS/MS analysis of THS on surface will only remove a tiny fraction of the materials, and can yet quantitatively detected these materials directly. However, whereas wiping-based methods can use dilution (to get back into a linear regime) when the mass-per-unit-area is too high to be in a linear range, in-situ DCBI cannot use that approach. For such situations, the area of the hole in the mask can easily be reduced, to detect higher mass concentration, up to 10,000 $\mu \mathrm{g} \mathrm{m}^{-2}$, so that for easy quantitative measurements in one go up to $10,000 \mu \mathrm{g} \mathrm{m}^{-2}$, a mask with two accurately specified holes of e.g. 2 and 6 $\mathrm{mm}$ should be used.

The results yield at least three observations: 1) The deposited amounts of nicotine and cotinine are highly surface-dependent. Likely, both the surface roughness and material characteristics like hydrophilic or hydrophobic will affect the adsorption of THS. Worrying is the high deposited amount found on rubber nipples. This high value reflects material properties: soft silicone rubber, with high THS contents per area versus polyethylene, with lower THS deposition per area. Surfaces with a high specific surface area and roughness, such as peach, filter paper, and cotton clothing, indeed display a high amount of THS. 2) Compared to conventional cigarettes, heat-not-burn (HNB) cigarettes yield lower cotinine amounts in their THS. This is in line with the lower concentration of cotinine found in SHS of HNB [39]. 3) Using this simple DCBI-MS/MS procedure, it is easily shown that basically all types of household materials absorb a significant amount of THS.

Next we aim to adapt the DCBI method to skin analysis. First, using pieces of filter paper of a specific area - as a crude model for skin - onto which dropped $2 \mu \mathrm{L}$ of a solution of $0.1 \mathrm{mg} \mathrm{mL}^{-1}$ nicotine and cotinine in either acetonitrile or water was placed, different DCBI desorption temperatures (instrument settings) from 50 to $300{ }^{\circ} \mathrm{C}$ were investigated, both with and without letting the sample left to dry. After the solution was dropped on the paper, the paper was placed under the corona beam to be analyzed. For solution in acetonitrile, the $\mathrm{S} / \mathrm{N}$ ratio slightly increased in going from $50^{\circ} \mathrm{C}$ to $300{ }^{\circ} \mathrm{C}$, namely from $370 \pm 40$ to $420 \pm$ 58 for nicotine $(n=5)$, and from $480 \pm 56$ to $579 \pm 67$ for cotinine ( $n=$ 5 ), likely because of the high volatility of acetonitrile. Filter paper wetted by aqueous solutions displayed similar $\mathrm{S} / \mathrm{N}$ ratios at temperatures higher than $250{ }^{\circ} \mathrm{C}(389 \pm 37$ for nicotine, and $420 \pm 50$ for cotinine, $(\mathrm{n}=5)$ ), but this decreased significantly when desorption temperature was reduced to $80{ }^{\circ} \mathrm{C}$ When the paper wetted with the aqueous solution was allowed to dry (ambient conditions, no heating), then desorption temperature could be decreased to $50{ }^{\circ} \mathrm{C}$, with $\mathrm{S} / \mathrm{N}$ ratios of nicotine ( $352 \pm 43, \mathrm{n}=5$ ) and cotinine ( $418 \pm 40, \mathrm{n}=5$ ) still similar to those obtained for the acetonitrile solution. In contrast to acetonitrile, the presence of significant amounts of water thus hampers the analysis, but trace amounts of water, as present in the ambient, do not appear to be detrimental.

Second, the success of this dried filter paper experiment prompted us to use the $50{ }^{\circ} \mathrm{C}$ DCBI desorption temperature analysis mode for human skin surface analysis. Here the set temperature was $50{ }^{\circ} \mathrm{C}$; this temperature would still be too high to endure for skin, but an infrared radiation thermometer indicated that the surface temperature at distances more than $2 \mathrm{~mm}$ away from the holder (electrode ring with holes, as visible in Fig. 4) was actually $\leq 35^{\circ} \mathrm{C}$ using this settings, i.e. fine for skin exposure. In addition, we checked for possible current injuries of DCBI, by measuring the charge current at various high voltages. Here we observed that even at $2.0 \mathrm{kV}$ and working in an ambient environment (relative humidity ambient, 80\%), the charge current was lower than 15 $\mu \mathrm{A}$, which is not harmful to humans. Therefore, DCBI with a set temperature of $50{ }^{\circ} \mathrm{C}$ can be used for the direct analysis of skin surfaces, and thus allows for a trivial analysis of e.g. finger tips (see Fig. 4).

Third, this now allows for the direct exposure of skin to THS by DCBIMS/MS, using these $50{ }^{\circ} \mathrm{C}$ settings. To this aim, for the real analysis, we wiped with a finger along THS-contaminated surfaces inside the THS 
a)

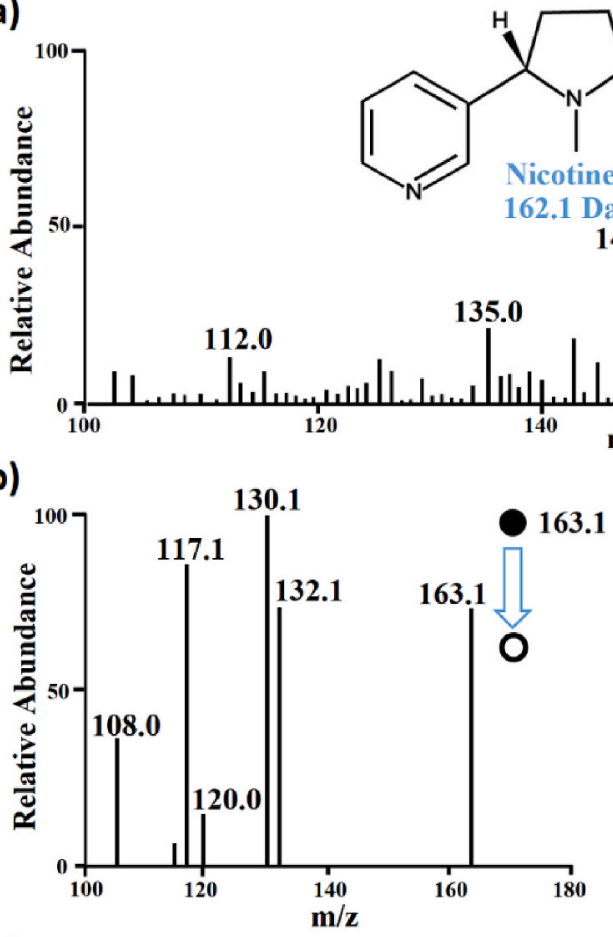

d)

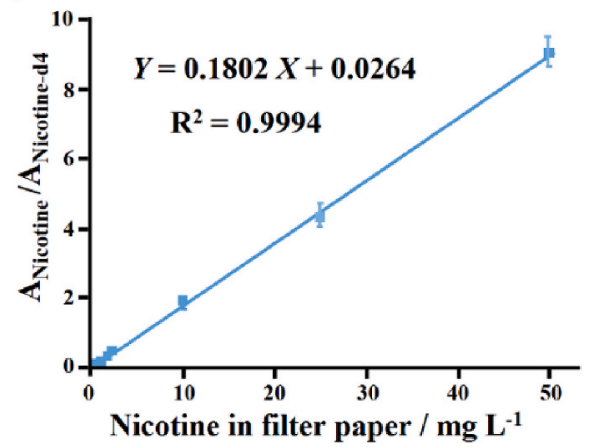

[Nicotine $+\mathbf{H}]^{+}$<smiles>CN1C(=O)C2CCCC21c1cccnc1</smiles>

190.1 176.1 Da
163.1

Fig. 3. Mass spectra obtained by DCBI analysis after filter paper exposure to smoke in the THS formation chamber, taking filter paper as example: (a) tobacco smoke exposure to filter paper; (b) MS/MS spectrum of protonated nicotine $(\mathrm{m} / \mathrm{z}$ 163.1) from (a); (c) MS/MS spectrum of protonated cotinine ( $\mathrm{m}$ / $z$ 177.1) from (a); (d) plot of signal peak area ratios of nicotine/nicotine- $\mathrm{d}_{4}$ against the concentration of nicotine in filter paper; (e) plot of signal peak area ratios of cotinine/ nicotine- $\mathrm{d}_{4}$ against the concentration of cotinine in filter paper.

c)

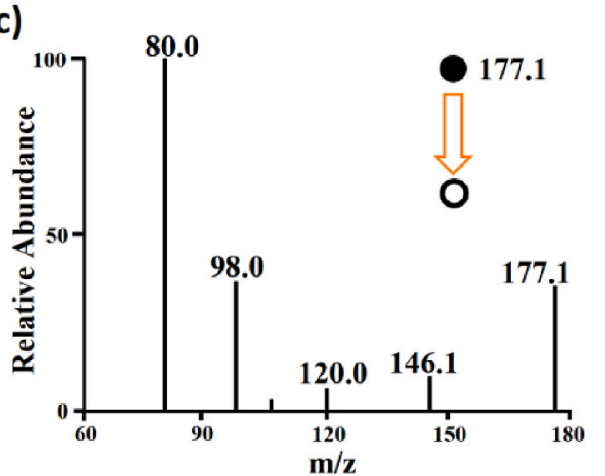

e)

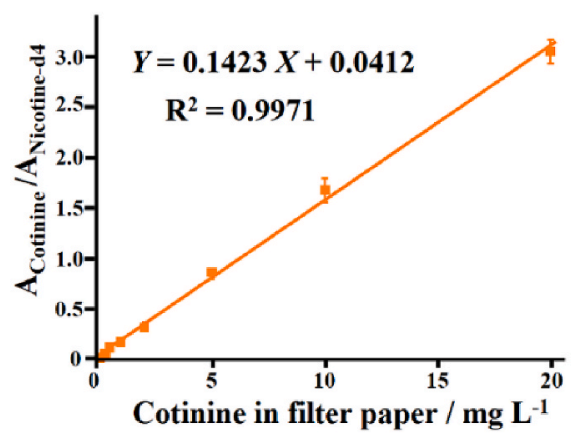

Table 1

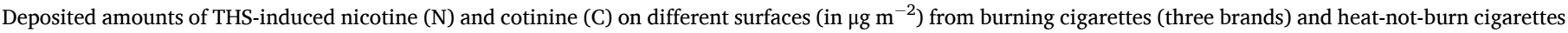
(two brands), as obtained from DCBI-MS/MS analysis. Error values are standard deviations ( $\mathrm{n}=3$ per experiment). All calculated value according to $0.28 \mathrm{~cm}{ }^{2}$.

\begin{tabular}{|c|c|c|c|c|c|c|}
\hline Sample & Compound & Burning Cigarette 1 & $\begin{array}{l}\text { Burning } \\
\text { Cigarette } 2\end{array}$ & $\begin{array}{l}\text { Burning } \\
\text { Cigarette } 3\end{array}$ & Heat-not-burn Cigarette 1 & $\begin{array}{l}\text { Heat-not-burn } \\
\text { Cigarette } 2\end{array}$ \\
\hline \multirow[t]{2}{*}{ Cherry tomato } & $\mathrm{N}$ & $38.9 \pm 1.2$ & $33.7 \pm 1.9$ & $34.8 \pm 0.7$ & $17.5 \pm 0.4$ & $15.8 \pm 0.5$ \\
\hline & $\mathrm{C}$ & ND & ND & ND & ND & ND \\
\hline \multirow[t]{2}{*}{ Apple } & $\mathrm{N}$ & $137.2 \pm 3.6$ & $143.7 \pm 2.0$ & $126.7 \pm 3.7$ & $111.5 \pm 4.1$ & $101.2 \pm 4.8$ \\
\hline & $\mathrm{C}$ & $2.2 \pm 0.3$ & $5.2 \pm 0.1$ & $4.1 \pm 0.1$ & ND & ND \\
\hline \multirow[t]{2}{*}{ Pear } & $\mathrm{N}$ & $224.7 \pm 4.8$ & $256.8 \pm 3.9$ & $202.4 \pm 2.1$ & $152.3 \pm 2.4$ & $143.1 \pm 1.7$ \\
\hline & $\mathrm{C}$ & $4.4 \pm 0.2$ & $9.3 \pm 0.4$ & $7.9 \pm 0.7$ & ND & ND \\
\hline \multirow[t]{2}{*}{ Peach } & $\mathrm{N}$ & $489.4 \pm 6.4$ & $465.3 \pm 9.2$ & $477.2 \pm 8.2$ & $234.7 \pm 2.9$ & $256.1 \pm 4.4$ \\
\hline & $\mathrm{C}$ & $10.4 \pm 0.6$ & $14.6 \pm 0.4$ & $9.5 \pm 1.1$ & $1.8 \pm 0.2$ & $2.7 \pm 0.2$ \\
\hline \multirow[t]{2}{*}{ Cotton clothing } & $\mathrm{N}$ & $782.3 \pm 5.6$ & $654.1 \pm 5.4$ & $733.4 \pm 8.8$ & $345.2 \pm 8.8$ & $219.5 \pm 2.8$ \\
\hline & $\mathrm{C}$ & $13.7 \pm 0.9$ & $10.6 \pm 1.0$ & $12.9 \pm 0.6$ & $3.9 \pm 0.2$ & $2.0 \pm 0.1$ \\
\hline \multirow[t]{2}{*}{ Plastic toy } & $\mathrm{N}$ & $32.1 \pm 1.5$ & $30.1 \pm 1.1$ & $37.2 \pm 1.6$ & $18.2 \pm 0.7$ & $19.3 \pm 1.6$ \\
\hline & $\mathrm{C}$ & ND & ND & ND & ND & ND \\
\hline \multirow[t]{2}{*}{ Infant milk bottle } & $\mathrm{N}$ & $112.4 \pm 2.4$ & $123.4 \pm 1.2$ & $110.7 \pm 2.4$ & $87.3 \pm 1.5$ & $67.1 \pm 1.9$ \\
\hline & $\mathrm{C}$ & $5.4 \pm 0.1$ & $4.7 \pm 0.2$ & $3.2 \pm 0.2$ & ND & ND \\
\hline \multirow[t]{2}{*}{ Rubber nipple } & $\mathrm{N}$ & $346.8 \pm 2.8$ & $311.2 \pm 1.9$ & $322.8 \pm 3.1$ & $198.4 \pm 2.0$ & $197.3 \pm 1.4$ \\
\hline & $\mathrm{C}$ & $20.7 \pm 0.4$ & $11.9 \pm 0.3$ & $3.5 \pm 0.1$ & $1.4 \pm 0.1$ & $2.2 \pm 0.1$ \\
\hline \multirow[t]{2}{*}{ Glass } & $\mathrm{N}$ & $27.9 \pm 1.2$ & $25.3 \pm 1.3$ & $25.7 \pm 0.9$ & $13.4 \pm 0.5$ & $13.2 \pm 0.6$ \\
\hline & $\mathrm{C}$ & $\mathrm{ND}$ & ND & $\mathrm{ND}$ & ND & ND \\
\hline \multirow[t]{2}{*}{ Filter paper } & $\mathrm{N}$ & $541.8 \pm 6.2$ & $487.0 \pm 9.0$ & $499.3 \pm 4.4$ & $311.6 \pm 3.6$ & $207.8 \pm 2.2$ \\
\hline & $\mathrm{C}$ & $12.1 \pm 1.2$ & $11.8 \pm 0.7$ & $15.5 \pm 0.9$ & $2.0 \pm 0.1$ & $2.2 \pm 0.1$ \\
\hline
\end{tabular}

Note: ND, not detected or below detection limits. 


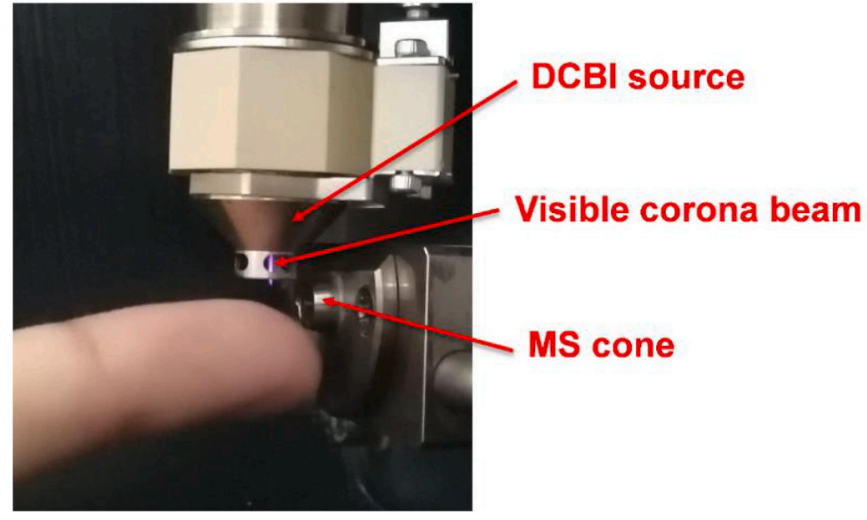

Fig. 4. Direct analysis of finger surface (of corresponding author B.C.) by DCBIMS/MS.

formation chamber. This included a plastic chair placed in there, and the inner side and top surfaces of the chamber itself. Next, we analyzed the thus potentially contaminated finger by DCBI-MS/MS after applying a 6 mm covering plastic mask and dropping $2 \mu \mathrm{L}$ of aqueous I.S. solution onto the finger. This yielded 180, 40, and $80( \pm 10) \mu \mathrm{g} \mathrm{m}^{-2}$ for nicotine $\left(\mathrm{LOD}=7.1 \mu \mathrm{g} \mathrm{m}^{-2}\right.$ ), where no nicotine was detected on a fingertip not exposed to THS. These numbers should only been taken as semiquantitative, given uncontrolled variables as the pressure and speed with which the surface was rubbed. However, the bottom line is clear: with DCBI-MS/MS one can directly measure the skin exposure to THS. This finding has significant ramifications for e.g. forensic and medical diagnostic studies, as it opens up the direct MS analysis of skin to study for the presence of illegal, harmful, or in contrast: medicinal compounds.

Since complex surfaces like skin could be analyzed easily, this prompted further investigations on rubber nipples, given the obvious higher risk (long-term contact between suckling baby and the material) and known high SHS-contamination level (Table 1). We simulated touching of and suckling on the nipple by extensive wiping of the area that is left open by the $6 \mathrm{~mm}$ mask with either dry or wet filter paper to simulate the transportation of THS from the rubber nipple surface to the baby, and measure the amount of nicotine and cotinine before and after the wiping. In both cases high mobility ratios were found: for a wet wiping $90 \pm 7 \%$ for nicotine and $86 \pm 5 \%$ for cotinine; for a dry wiping $73 \pm 8 \%$ for nicotine, $61 \pm 7 \%$ for cotinine. In other words: in both cases most of the nicotine and cotinine is removed from the rubber nipples, and thus will likely end up in the baby. Given the high THS contamination of such soft and porous materials in the first place, this high mobility ratio points to significant THS-induced risks.

\subsection{Biomarkers of THS detection in urine by DCBI}

Nicotine and its metabolite cotinine are the most specific of the commonly used biomarkers for tobacco-smoke exposure, as analyzed in serum, urine, and saliva [28,40,41]. Especially urine is of interest, given its non-invasive sampling and direct indication of the concentration of nicotine and cotinine in the kidney. Typically, HPLC-MS/MS or GC-MS are used [42,43], but either method is time-consuming and allows only low throughput. A paper spray (PS) MS method was developed as a high-throughput method for the quantification of nicotine and cotinine at the air-liquid interface (ALI) on human tracheobronchial epithelial cell cultures exposed to tobacco smoke [23], but this PS-MS method was not applied to analyze body fluids with a high salt background. Because DCBI relies on gas phase ionization, similar to APCI or DART, the effect of salts in the sample on the ionization is small. In addition, since the thermal desorption is very fast, and the $\mathrm{S} / \mathrm{N}$ is better than PS-MS, this prompted us to investigate whether DCBI could be a suitable method for the direct analysis of nicotine and cotinine in biological fluids with a high salt concentration, specifically urine.

For the evaluation of salt concentration effect on the overall outcome, on the LODs and on linearity of the nicotine and cotinine signals, urine was mixed $(1: 1, \mathrm{v} / \mathrm{v})$ with different concentrations $(0.5 \%$, $1.0 \%$, and $5.0 \%) \mathrm{NaCl}$ aqueous solutions, and investigated. When $5 \mu \mathrm{L}$ of solution was directly dropped at the tip of sampling capillary and analyzed by DCBI-MS/MS, it became evident that increasing salt concentrations generically decreased the response of nicotine and cotinine. This effect could be counteracted by increasing the desorption temperature. When the temperature was higher than $300{ }^{\circ} \mathrm{C}$, the LODs of nicotine were 500,800 and $2000 \mathrm{ng} \mathrm{mL}^{-1}$ for urine with the $0.5 \%, 1.0 \%$, and $5.0 \% \mathrm{NaCl}$ solutions, respectively. Diluting these samples a further four-fold dilution by acetonitrile, decreased the LODs even further, to 50,50 , and $1000 \mathrm{ng} \mathrm{mL}{ }^{-1}$. Specifically, the observation that simple dilution of urine with a water/acetonitrile mixture yields such LODs is of interest for further studies: the LODs are, of course, significantly higher than obtainable via SPE-HPLC-MS/MS, but the minimal pretreatment in our case more easily allows for sampling on a larger scale in cases where such ultra-low LODs are not needed.

Calibration curves of nicotine and cotinine in urine were constructed using DCBI-MS/MS (see Fig. 5). This shows excellent linear relation $\left(\mathrm{R}^{2}\right.$ $=0.9982$ for nicotine, concentration range $0.1-50 \mathrm{mg} \mathrm{L}^{-1}$ and $\mathrm{R}^{2}=$ 0.9968 for cotinine, concentration range $0.1-20 \mathrm{mg} \mathrm{L}^{-1}$ ) by using internal standard solution (nicotine- $\mathrm{d}_{4}, 1.0 \mathrm{mg} \mathrm{L}^{-1}$ ). Using this approach urine samples from 52 (random) adult Hunan citizens were obtained and analyzed, to investigate the precision of our method. This yielded 4 samples without nicotine and cotinine (lower than LODs), but nicotine and cotinine were clearly detected in all other 48 samples. In these, the nicotine and cotinine level ranges were $0.3-17 \mathrm{mg} \mathrm{L}^{-1}$ with $2.4-5.1 \%$ of RSD range ( $\mathrm{n}=3$ ), and $0.2-16.2 \mathrm{mg} \mathrm{L}^{-1}$ with $3.1-5.7 \%$ of RSD range ( $=3$ ), respectively. Most relevant here was the low standard deviation (max $\pm 5 \%$; typically $\pm 2 \%$ ) over the entire range of concentrations, showing the potential value of this technique in large-scale studies in which the LODs is not limiting, making DCBI-MS/MS a useful method to facilitate distinguishing between users and non-users of certain drugs (ranging from illegal to medicinal compounds).

The LODs is limiting in urine analysis of young children. Yet, these are among the most vulnerable to THS, and the analysis shown above shows that transfer of THS via e.g. suckling might be significant. Previous work on SHS exposure has shown cotinine levels that are up to 17 times higher for babies with parents that were smoking in the home, than for babies that had no smokers in the home [44,45], with relevant cotinine levels of $0.3-1.1 \mathrm{ng} \mathrm{mL}^{-1}$ for saliva and urine. Therefore any analytical method should have a LODs lower than $0.1 \mathrm{ng} \mathrm{mL}^{-1}$ to be meaningful for such studies. The current, direct DCBI-MS/MS method, is limited in this regard, but a DCBI-based method would still be of significant interest as it would be fast compared with HPLC-MS/MS and GC-MS.

To investigate the possibility to use DCBI for such high-sensitivity analysis, we used dispersive liquid-liquid micro-extraction (DLLME) to enrich two THS biomarkers (nicotine and cotinine) in urine of six newborn' and of eight 3-9 month old infants (specific samples preparation see section 2.4). Calibration curves yielded a clearly linear response, and the LODs of nicotine and cotinine in urine were observed to be $0.01 \mathrm{ng} \mathrm{mL}^{-1}$ and $0.02 \mathrm{ng} \mathrm{mL}^{-1}$, respectively. Such LODs certainly allow the use of DCBI-analysis in sample analysis from newborns and slightly older babies. Therefore we analyzed the urine of six newborns and of eight 3 9-month old babies. There was no detection of nicotine and cotinine in any of the newborns and in the urine of six of the babies (i.e., concentrations lower than LODs). However, the concentrations of nicotine and cotinine in two babies' urine (age: 9 and 5 months, respectively) were $0.3 \mathrm{ng} \mathrm{mL}^{-1}$ and $0.1 \mathrm{ng} \mathrm{mL}^{-1}$ (baby 1 ), and $0.5 \mathrm{ng}$ $\mathrm{mL}^{-1}$ and $0.2 \mathrm{ng} \mathrm{mL}^{-1}$ (baby 2), respectively. These data show that DCBI-based analyses can clearly confirms the presence of SHS and THSbased compounds, even in little children, while they also show the harm 


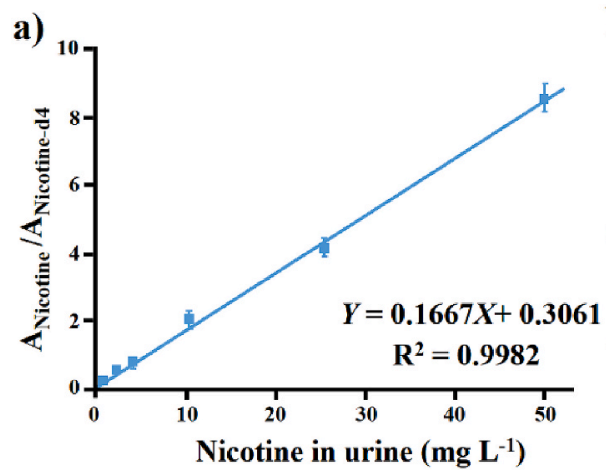

b)

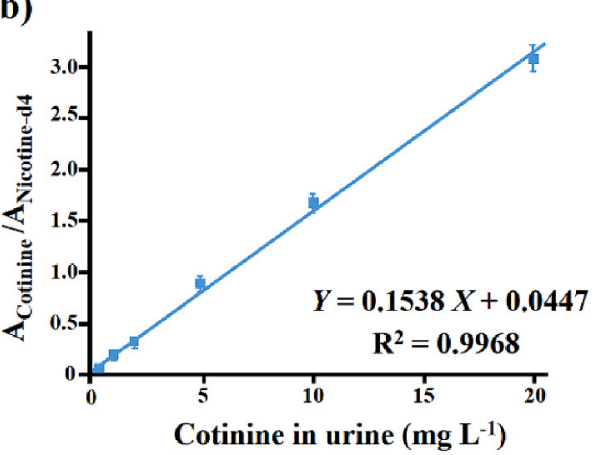

Fig. 5. Calibration curves of nicotine (a) and cotinine (b) in urine. Error bars represent standard deviation of triplicate measurements at each concentration.

to really young people of smoking in their environment.

\subsection{Direct and in-situ monitoring of TSNAs}

Nicotine can, especially on warm surfaces, react with nitrous acid (HONO) to produce nitrosamines specific to tobacco, TSNAs, such as: NNA, NNK and NNN, which are known to be highly mutagenic and carcinogenic [6]. Especially in a surface-bound state, nicotine displays a high reactivity towards HONO, as investigated by reported methods [6, 46-48].

For the formation of TSNAs, there are two environmentally relevant modes. The first involves the slow formation on surfaces at room temperature reactions, during and after which TSNAs remain in THS deposits. The second one is the rapid reaction of THS components with HONO in high-temperature reactions, e.g. cigarette burning or the heating of food contaminated by THS. Here, we investigated the first, and collected 31 dust samples in 'smoking allowed'-hotel rooms from a variety of locations (cabinet tops, under sofas, on window sills and inside ashtrays). Experiments were performed in positive ion mode and gas temperature $300{ }^{\circ} \mathrm{C}$, and the MS/MS characteristic ion pairs of NNA, NNK and NNN were monitored respectively. Then the calibration curves were constructed using the signal peak area ratio $(Y)$ of target ion to internal standard ion to the concentration of target ion $(X)$. In all cases good linearity was observed $\left(\mathrm{R}^{2}>0.992\right)$ in the range $0.1-50 \mathrm{mg} \mathrm{L}^{-1}$, and both NNA, NNK and NNN characteristic LODs were found to be 0.02

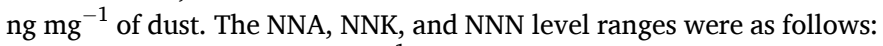

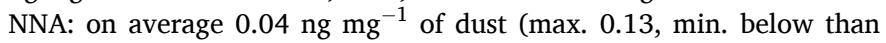
LODs) with $9.4-15.3 \%$ of RSD range $(\mathrm{n}=3)$; NNK: on average $1.19 \mathrm{ng}$ $\mathrm{mg}^{-1}$ of dust (max. 3.72, min. 0.32$)$ with $4.2-7.3 \%$ of RSD range $(\mathrm{n}=3)$; NNN: on average $0.64 \mathrm{ng} \mathrm{mg}^{-1}$ (max. 1.20 , min. 0.23 ) with $5.7-7.8 \%$ of RSD range $(n=3)$, respectively. In this experiment, TSNAs of dust sample were directly detected without pretreatment process by DCBIMS/MS. Although the precision of this method was somewhat limited, the high speed of this analysis (just 2-4 min for whole procedure from dust sampling to analysis) again demonstrates the power of DCBI analyses, and confirms the presence of HONO reaction products on everyday surfaces in smokers' rooms.

\section{Conclusions}

Direct Corona Beam Ionization MS/MS allows for the direct and quantitative analysis of Third-Hand Smoke and THS-derived products on both unnatural surfaces (rubber nipple, cotton clothing, glass etc.), on finger tips and in urine. The DCBI analysis can also, without harm, be extended to skins that were exposed to a wide range of agents that are either unintendedly present (like THS, remnants of drug use) or are in fact intended to be there (e.g., medication). Given the flexibility and ease-of-use of this approach, DCBI analysis is not only very meaningful for the high-throughput evaluation of THS exposure and THS reaction products discovery, but also to other high-throughput exposure studies.

\section{Author statement}

Ke Min: Methodology, Investigation, Writing- Original draft preparation, Ping Guo: Data curation, Software, Visualization, Dongying Chen: Funding acquisition, Si Huang, Wei Luo: Supervision administration, Ming Ma: Validation, Bo Chen: Writing- Reviewing and Editing, Conceptualization, Shouzhuo Yao: Term, Conceptualization, Han Zuilhof: Writing- Reviewing

\section{Declaration of competing interest}

The authors declare that they have no known competing financial interests or personal relationships that could have appeared to influence the work reported in this paper.

\section{Acknowledgements}

The authors acknowledge support from the National Natural Science Foundation of China (21775040, 21775041, 21575040), the Aid Program for S\&T innovation research team in higher education institutions, the construction program of key disciplines of Hunan Province (2015JC1001), the Hunan Province 100 experts project, and Wageningen University.

\section{Appendix A. Supplementary data}

Supplementary data to this article can be found online at https://doi. org/10.1016/j.talanta.2020.121330.

\section{References}

[1] L. Szabo, Babies May Absorb Smoke Residue in Home. Usa Today, 2006. https://u satoday30.usatoday.com/news/health/2006-08-06-thirdhand-smoke-usat_x.htm.

[2] C. Protano, M. Vitali, The new danger of thirdhand smoke: why passive smoking does not stop at secondhand smoke, Environ. Health Perspect. 119 (2011) A422.

[3] P. Jacob 3rd, N.L. Benowitz, H. Destaillats, L. Gundel, B. Hang, M. Martins-Green, G.E. Matt, P.J. Quintana, J.M. Samet, S.F. Schick, P. Talbot, N.J. Aquilina, M. F. Hovell, J.H. Mao, T.P. Whitehead, Thirdhand smoke: new evidence, challenges, and future directions, Chem. Res. Toxicol. 30 (2017) 270-294.

[4] G.E. Matt, P.J. Quintana, H. Destaillats, L.A. Gundel, M. Sleiman, B.C. Singer, P. Jacob, N. Benowitz, J.P. Winickoff, V. Rehan, P. Talbot, S. Schick, J. Samet, Y. Wang, B. Hang, M. Martins-Green, J.F. Pankow, M.F. Hovell, Thirdhand tobacco smoke: emerging evidence and arguments for a multidisciplinary research agenda, Environ. Health Perspect. 119 (2011) 1218-1226.

[5] A. Díez-Izquierdo, P. Cassanello-Peñarroya, C. Lidón-Moyano, N. MatillaSantander, A. Balaguer, J.M. Martínez-Sánchez, Update on thirdhand smoke: a comprehensive systematic review, Environ. Res. 167 (2018) 341-371.

[6] M. Sleiman, L.A. Gundel, J.F. Pankow, P. Jacob 3rd, B.C. Singer, H. Destaillats, Formation of carcinogens indoors by surface-mediated reactions of nicotine with nitrous acid, leading to potential thirdhand smoke hazards, Proc. Natl. Acad. Sci. U. S.A. 107 (2010) 6576-6581.

[7] L.M. Petrick, A. Svidovsky, Y. Dubowski, Thirdhand smoke: heterogeneous oxidation of nicotine and secondary aerosol formation in the indoor environment, Environ. Sci. Technol. 45 (2011) 328-333.

[8] M. Sleiman, H. Destaillats, J.D. Smith, C.-L. Liu, M. Ahmed, K.R. Wilson, L. A. Gundel, Secondary organic aerosol formation from ozone-initiated reactions 
with nicotine and secondhand tobacco smoke, Atmos. Environ. 44 (2010) 4191-4198.

[9] M.L. Goniewicz, L. Lee, Electronic cigarettes are a source of thirdhand exposure to nicotine, Nicotine Tob, Res. 17 (2015) 256-258.

[10] S.F. Schick, K.F. Farraro, C. Perrino, M. Sleiman, G. van de Vossenberg, M.P. Trinh, S.K. Hammond, B.M. Jenkins, J. Balmes, Thirdhand cigarette smoke in an experimental chamber: evidence of surface deposition of nicotine, nitrosamines and polycyclic aromatic hydrocarbons and de novo formation of NNK, Tobac. Contr. 23 (2014) 152-159.

[11] D.K. Farmer, Analytical challenges and opportunities for indoor air chemistry field studies, Anal. Chem. 91 (2019) 3761-3767.

[12] Z. Takáts, J.M. Wiseman, B. Gologan, R.G. Cooks, Mass spectrometry sampling under ambient conditions with desorption electrospray ionization, Science 306 (2004) 471.

[13] D. Gahtory, R. Sen, A.R. Kuzmyn, J. Escorihuela, H. Zuilhof, Strain-promoted cycloaddition of cyclopropenes with o-quinones: a rapid click reaction, Angew. Chem. Int. Ed. Engl. 57 (2018) 10118-10122.

[14] D. Gahtory, R. Sen, S. Pujari, S. Li, Q. Zheng, J.E. Moses, K.B. Sharpless, H. Zuilhof, Quantitative and orthogonal formation and reactivity of SuFEx platforms, Chem. Eur J. 24 (2018) 10550-10556.

[15] D. Gahtory, R. Sen, M.M.J. Smulders, H. Zuilhof, Surface-bound quadruple Hbonded dimers: formation and exchange kinetics, Faraday Discuss 204 (2017) 383-394.

[16] F.A. Van Geenen, M.C. Franssen, A.H. Schotman, H. Zuilhof, M.W.F. Nielen, Ambient characterization of synthetic fibers by laser ablation electrospray ionization mass spectrometry, Anal. Chem. 89 (2017) 4031-4037.

[17] C.L. Feider, A. Krieger, R.J. DeHoog, L.S. Eberlin, Ambient ionization mass spectrometry: recent developments and applications, Anal. Chem. 91 (2019) 4266-4290.

[18] R.K. Manova, S. Joshi, A. Debrassi, N.S. Bhairamadgi, E. Roeven, J. Gagnon, M. N. Tahir, F.W. Claassen, L.M. Scheres, T. Wennekes, K. Schroen, T.A. van Beek, H. Zuilhof, M.W.F. Nielen, Ambient surface analysis of organic monolayers using direct analysis in real time Orbitrap mass spectrometry, Anal. Chem. 86 (2014) 2403-2411.

[19] R. Sen, J. Escorihuela, F. van Delft, H. Zuilhof, Rapid and complete surface modification with strain-promoted oxidation-controlled cyclooctyne-1,2-quinone cycloaddition (SPOCQ), Angew. Chem. Int. Ed. Engl. 56 (2017) 3299-3303.

[20] B. Spengler, Mass spectrometry imaging of biomolecular information, Anal. Chem. 87 (2015) 64-82.

[21] F. van Geenen, M.C.R. Franssen, V. Miikkulainen, M. Ritala, H. Zuilhof, R. Kostiainen, M.W.F. Nielen, TiO2 photocatalyzed oxidation of drugs studied by laser ablation electrospray ionization mass spectrometry, J. Am. Soc. Mass Spectrom. 30 (2019) 639-646.

[22] M. Schrage, Y. Shen, F.W. Claassen, H. Zuilhof, M.W. Nielen, B. Chen, T.A. van Beek, Rapid and simple neurotoxin-based distinction of Chinese and Japanese star anise by direct plant spray mass spectrometry, J. Chromatogr., A 1317 (2013) $246-253$.

[23] J.E. Keating, J.T. Minges, S.H. Randell, G.L. Glish, Paper spray mass spectrometry for high-throughput quantification of nicotine and cotinine, Anal. Methods. 10 (2018) 46-50.

[24] Á. Kuki, L. Nagy, T. Nagy, M. Zsuga, S. Kéki, Detection of nicotine as an indicator of tobacco smoke by direct analysis in real time (DART) tandem mass spectrometry, Atmos. Environ. 100 (2015) 74-77.

[25] S. Torres, C. Merino, B. Paton, X. Correig, N. Ramirez, Biomarkers of exposure to secondhand and thirdhand tobacco smoke: recent advances and future perspectives, Int. J. Environ. Res. Publ. Health 15 (2018) 2693.

[26] T.F. Northrup, A.M. Khan, P. Jacob 3rd, N.L. Benowitz, E. Hoh, M.F. Hovell, G. E. Matt, A.L. Stotts, Thirdhand smoke contamination in hospital settings: assessing exposure risk for vulnerable paediatric patients, Tobac. Contr. 25 (2016) 619-623.

[27] G.E. Matt, P.J.E. Quintana, J.M. Zakarian, E. Hoh, M.F. Hovell, M. MahabeeGittens, K. Watanabe, K. Datuin, C. Vue, D.A. Chatfield, When smokers quit: exposure to nicotine and carcinogens persists from thirdhand smoke pollution, Tobac. Contr. 26 (2016) 548-556.

[28] G.E. Matt, P.J. Quintana, A.L. Fortmann, J.M. Zakarian, V.E. Galaviz, D. A. Chatfield, E. Hoh, M.F. Hovell, C. Winston, Thirdhand smoke and exposure in California hotels: non-smoking rooms fail to protect non-smoking hotel guests from tobacco smoke exposure, Tobac. Contr. 23 (2014) 264-272.
[29] H. Wang, W. Sun, J. Zhang, X. Yang, T. Lin, L. Ding, Desorption corona beam ionization source for mass spectrometry, Analyst 135 (2010) 688-695.

[30] Y.Q. Huang, J.Q. You, J. Zhang, W. Sun, L. Ding, Y.Q. Feng, Coupling frontal elution paper chromatography with desorption corona beam ionization mass spectrometry for rapid analysis of chlorphenamine in herbal medicines and dietary supplements, J. Chromatogr., A 1218 (2011) 7371-7376.

[31] H. Wang, Z. Fei, Z. Li, R. Xing, Z. Liu, Y. Zhang, H. Ding, Coupling laser desorption with corona beam ionization for ambient mass spectrometric analysis of solution and powder samples, Talanta 179 (2018) 364-368.

[32] Y. Hou, T. Wu, Y. Liu, H. Wang, Y. Chen, B. Chen, W. Sun, Direct analysis of quaternary alkaloids by in situ reactive desorption corona beam ionization MS, Analyst 139 (2014) 5185-5191.

[33] D. Chen, H.B. Zheng, Y.Q. Huang, Y.N. Hu, Q.W. Yu, B.F. Yuan, Y.Q. Feng, Magnetic solid phase extraction coupled with desorption corona beam ionizationmass spectrometry for rapid analysis of antidepressants in human body fluids, Analyst 140 (2015) 5662-5670.

[34] H. Wang, Y. Wu, B. Guo, W. Sun, L. Ding, B. Chen, Quantification of low-polar small molecules using room temperature ionic liquids matrix-assisted desorption corona beam ionization, Analyst 137 (2012) 3982-3988.

[35] H. Wang, Y. Wu, Y. Zhao, W. Sun, L. Ding, B. Guo, B. Chen, Rapid screening of illicit additives in weight loss dietary supplements with desorption corona beam ionisation (DCBI) mass spectrometry, Food Addit. Contam. 29 (2012) 1194-1201.

[36] X. Li, H. Wang, W. Sun, L. Ding, Desorption corona beam ionization coupled with a poly (dimethylsiloxane) substrate: broadening the application of ambient ionization for water samples, Anal. Chem. 82 (2010) 9188-9193.

[37] W. Du, L.J. Tang, J.H. Wen, K.J. Zhong, J.H. Jiang, H. Wang, B. Chen, R.Q. Yu, Desorption corona beam ionisation (DCBI) mass spectrometry for in-situ analysis of adsorbed phenol in cigarette acetate fiber filter, Talanta 131 (2015) 499-504.

[38] G. Xu, B. Chen, H. Wang, Y. Chen, L. Ding, S. Yao, Detection of intermediates in Leuckart reaction by desorption corona beam ionization ambient mass spectrometry, Anal. Methods. 4 (2012) 3098-3101.

[39] Y.Q. Chen, Y. Hong, L.J. Zeng, Y.X. Wen, C.R. Luo, Determination of release behaviour of flue-cured tobacco by simulated pyrolysis technology, Food Mach. (Ch.) 31 (2015) 19-31.

[40] K.M. Wilson, J.D. Klein, A.K. Blumkin, M. Gottlieb, J.P. Winickoff, Tobacco-smoke exposure in children who live in multiunit housing, Pediatrics 127 (2011) 85-92.

[41] P. Jacob 3rd, M.L. Goniewicz, C.M. Havel, S.F. Schick, N.L. Benowitz, Nicotelline: a proposed biomarker and environmental tracer for particulate matter derived from tobacco smoke, Chem. Res. Toxicol. 26 (2013) 1615-1631.

[42] M.A. Kaisar, R.R. Kallem, R.K. Sajja, A.E. Sifat, L. Cucullo, A convenient UHPLCMS/MS method for routine monitoring of plasma and brain levels of nicotine and cotinine as a tool to validate newly developed preclinical smoking model in mouse, BMC Neurosci. 18 (2017) 71.

[43] M.N. Tzatzarakis, C.I. Vardavas, I. Terzi, M. Kavalakis, M. Kokkinakis, J. Liesivuori, A.M. Tsatsakis, Hair nicotine/cotinine concentrations as a method of monitoring exposure to tobacco smoke among infants and adults, Hum. Exp. Toxicol. 31 (2012) 258-265.

[44] M.P. Wang, Y.N. Suen, B.Y. Wong, W.H. Li, D.S. Koh, T.H. Lam, S.S. Chan, Paternal smoking and maternal protective behaviors at home on infant's saliva cotinine levels, Pediatr. Res. 83 (2018) 936-942.

[45] S. Willers, A. Axmon, C. Feyerabend, J. Nielsen, G. Skarping, S. Skerfving, Assessment of environmental tobacco smoke exposure in children with asthmatic symptoms by questionnaire and cotinine concentrations in plasma, saliva, and urine, J. Clin. Epidemiol. 53 (2000) 715-721.

[46] S.S. Hecht, C.-H.B. Chen, R.M. Ornaf, E. Jacobs, J.D. Adams, D. Hoffmann, Chemical studies on tobacco smoke. 52. Reaction of nicotine and sodium nitrite: formation of nitrosamines and fragmentation of the pyrrolidine ring, J. Org. Chem. 43 (1978) 72-76.

[47] E. Konstantinou, F. Fotopoulou, A. Drosos, N. Dimakopoulou, Z. Zagoriti, A. Niarchos, D. Makrynioti, D. Kouretas, K. Farsalinos, G. Lagoumintzis, K. Poulas, Tobacco-specific nitrosamines: a literature review, Food Chem. Toxicol. 118 (2018) 198-203.

[48] K.D. Brunnemann, L. Genoble, D. Hoffmann, Identification and analysis of a new tobacco-specific iV-nitrosamine, 4-(methylnitrosamino)-4-(3-pyridyl)-1-butanol, Carcinogenesis 8 (1987) 465-469. 